\title{
Electoral College and Direct Popular Vote for Multi-Candidate Election
}

Liang Chen

Ichen@ieee.org;chen.liang.97@gmail.com

\author{
University of Northern British Columbia \\ 3333 University Way, Prince George, \\ BC, Canada V2N 4 Z9
}

\begin{abstract}
We introduce a stability analysis model for multi-candidate regional and national voting schemes (aka Electoral College and Direct Popular Vote, respectively), which can be expressed as the a posteriori probability that a winning candidate will continue to be chosen after the system is subjected to noise. The model shows, in most situations, that regional voting is more stable than national voting; that the stability of regional voting increases as the size of the subdivided regions decreases, up to a certain level, and then the stability starts to decrease approaching the stability of national voting as the region size approaches the original unit cell size; and that the stability of regional voting approaches that of national voting in the two extremities as the regional size increases to the original national voting size or decreases to the unit cell size. It also shows, for the special situation of homogeneous noise dominance with negligibly small amount of inhomogeneous noise, that national voting is surprisingly more stable than regional voting. The theory is evaluated by face recognition experiments on FERET and Yale Face datasets where exceptional improvements have been achieved.
\end{abstract}

\section{Introduction}

The objective of typical pattern recognition problems, such as a face recognition problem, is to find a computable mapping $f: X \rightarrow D_{I}$, where $D_{I}=\left\{I_{1}, I_{2}, \cdots, I_{n}\right\}$ represents a set of known objects, which may be called the gallery, or the database; such that for an input $\mathscr{O}$, $f(\mathscr{O})$, should be its identity. The performance of a pattern recognition algorithm or function, $f$, can be measured by its accuracy on a set of objects $X$ which is not included in the database / gallery $D_{I}$.

There is a philosophical problem though, before we discuss accuracy: while an object $\mathscr{O}$ is neither identical to $I_{i}$ nor $I_{j}$ in the gallery, what makes humans think that it actually is object $I_{i}$ but not object $I_{j}$ ? For human face recognition, for example, although according to certain similarity measurements, the similarity of $\mathscr{O}$ and $I_{i}$ is greater than $\mathscr{O}$ and $I_{j}$; do these measurements match the real psychological process of humans recognizing a face? Mathematics, which is remarkably effective in explaining the world, does not work to explain the psychological processes of the human mind itself [ $\square$, p.2]. To the author's point of view, it is bound to fail if attempting to mathematically reason the humans' face recognition process.

Following this argument, we define the resistance of the system to noise as the performance metric for a recognition system. We do not argue, in the above example, if $\mathscr{O}$ should be $I_{i}$ or $I_{j}$, we only request that the conclusion be resistant to environmental change. That 
is, we concentrate on checking if $f\left(\mathscr{O}^{\prime}\right)$ is still $I_{x}$, when $\mathscr{O}^{\prime}$ is transformed from $\mathscr{O}$ due to a certain level of environmental change and $f(\mathscr{O})=I_{x}$.

In this paper, we assume that the 'transformations' making an object $\mathscr{O}^{\prime}$ 'look' different from $\mathscr{O}$ are caused by 'pollution' or 'noise' on $\mathscr{O}$. Thereafter, we measure the 'level' of transformation by the 'amount' of change caused by noise; we measure the performance of a pattern recognition algorithm $f$ by the probability that $f\left(\mathscr{O}^{\prime}\right)=f(\mathscr{O})$ ) when $\mathscr{O}$ is transformed to $\mathscr{O}$ with a fixed 'amount' of changes caused by noise. Here, 'typical' means that the object $\mathscr{O}$ is close to the boundary of at least two objects in $D_{I}$ in terms of similarity values.

One immediate question arises: How does stability affect the accuracy of a pattern recognition system? When a "good" system implies that it is able to recognize a pattern after a "great" amount of "change", improving the stability of a system means improving the chance that the system can recognize an object which differs a lot from its original appearance - which will be reflected in its improved accuracy.

We focus on the stability study on two categories of pattern recognition schemes, namely, Direct Popular Match/Vote and Electoral College Match/Vote. To simplify the description, we assume here that all the patterns, including input patterns and the patterns in the gallery, are of equal rectangular size. We only consider two types of noise: Inhomogeneous (concentrated) noise that appears in clusters or blocks and homogeneous noise that shows in the form of salt-and-pepper ${ }^{1}$.

Direct Popular Vote and Electoral College are familiar to most people in everyday life. In the context of pattern recognition, in direct popular vote, we select the winner from among candidate patterns (in the gallery) directly by a simple majority of the entire vote population/all the pixels in the rectangle area. The Electoral College on the other hand, involves a more complex process whereby winners are determined by the majority of the winning regions/windows into which the entire national voting district is divided.

The direct pixel-by-pixel/cell-to-cell matching has to be avoided in most practical applications in the pattern-processing field due to the known registration problem, the direct popular vote / national matching scheme recognizes objects commonly based on global features extracted from an entire image and the Electoral College / regional matching scheme commonly uses two steps: the first step usually makes tentative local decisions based on local features all extracted from the local areas, and the second step obtains a final conclusion by integrating the entire local decisions.

Some progress has been made in the stability analysis of the two categories of pattern recognition schemes for two-candidate models since 1999 ([ $[\mathrm{Q}, \mathrm{\theta}, \mathrm{\theta}, \mathrm{\theta}]$ ). The stability measurement in the these papers defines the largest amount of noise contaminated votes that a vote system can accommodate so as to guarantee that the original winner of the vote without pollution still has a higher probability of winning than the other(s).

In most of situations, however, when the number of candidates is a very big number, it is more important for a candidate to have the highest possible probability of winning, which might not be higher than some other candidates, than continuing to be the leading candidate which is meant to be the one having a highest probability of winning among all candidates. This means that, when many candidates are involved, in view of stability of pattern recognition, we should examine the probability of leading candidate continuing to be the winner rather than just being the one with highest probability of winning - therefore, the stability measument in previous papers [ $[\boldsymbol{\theta}, \boldsymbol{\theta}]$ must be abandoned.

\footnotetext{
${ }^{1}$ In the context of image processing, inhomogeneous (concentrated) noise applies to damaged or polluted images due to ink blotting, leaking sunlight through sunshade, carriers, or electric noise at a certain time periods [వ], homogeneous noise is isolated and scattered randomly over discrete "points" of the entire image.
} 
In this paper, we develop a new model to study the performance of national and regional voting systems for multiple candidates / selecitons by measuring stability of a voting system as the posteriori probability that the winning candidate will be chosen again as the winner in environments containing both homogeneous and inhomogeneous noise. We will compare, under the same amount of noise, the probabilities that the voting systems keep the original candidate selections. The theory will be evaluated by face recognition experiments on FERET and Yale Face dataset, where impressive performances have been achieved.

The rest of the paper is constructed as follows: We introduce the basic terms used in Section 2. The main theoretical results regarding stability are given in Section 3. The experiments on face recognition which verify the theory are provided in 4 . The discussion and further work are given in Section 5. The program codes for one set of the experiments are included in "Supplementary Material", and also available at http: / / web . unbc.ca / $\sim$ chenl/DataCode.html.

\section{Basic Model \& Assumption}

For a multi-candidate election, there are many different ways of implementing the winner selection. Consider the two typical examples here: the best-of-all strategy and the pair-wisebest strategy. When confronted with the selection of a candidate, the best-of-all strategy allows a voter to choose their best choice from among all the candidates, and the candidate getting the largest number of votes is the winner. The pair-wise-best strategy allows voters to provide preference between each pair of candidates, and the candidate who gets the larger number of votes between any pair of candidates is selected as the winner. Both of these two strategies have advantages and disadvantages. In this paper, we choose the "pair-wisebest" strategy in selecting the winner, essentially because it can simplify the mathematical derivations and expressions.

To facilitate the analysis, we assume an equilibrium state that in the absence of noise, a candidate $A$ is a dominant front running candidate with $m$ other candidates; $B_{i}, i=1,2, \cdots, m$, form a group of candidates following closely behind candidate $A$. Consequently, our model can be described as follows:

1. The nation is represented by a rectangular area comprising $l \times w=N(l$ and $w$ being positive integers) cells, where a cell is the basic unit of votes; i.e., 1 cell comprises 1 vote.

2. We consider a multi-candidate model with $m+1$ candidates $A, B_{1}, B_{2}, \ldots, B_{m}$, but in comparison with any candidate $B_{i}$, candidate $A$ has slightly more supporters.

3. The pair-wise selection strategy allows all voters to select a pair of candidates $A$ and any of $B_{i}$. Let $\alpha$ and $\beta$ denote the probability of a vote choosing $A$ and $B_{i}$ respectively.

4. For regional voting purposes, the rectangular nation is divided into equal-shaped rectangles, called regions of size $r_{l} \times r_{w}\left(r_{l}\right.$ and $r_{w}$, which are positive integers), where $l$ and $w$ are divisible by $r_{l}$ and $r_{w}$ independently.

5. National voting is implemented over the entire nation, and a winner in the national voting is decided by a simple majority of the votes throughout the nation. A winner in the regional voting is decided by applying the "winner-take-all" principle on winning regions, namely, by a majority of the winning regions, where the winner of each region is determined by a simple majority of votes within the region.

6. Noise is defined as factors affecting environments that cause a change of the voting result; when subjected to noise, the votes of some of the cells will undergo a change from $A$ to $B_{i}$, some from $B_{i}$ to $A$, and others may remain unchanged. The noise that causes votes to change from $A$ to $B_{i}$ (or $B_{i}$ to $A$ ) is called anti- $A$ noise (or anti- $B$ noise, respectively). A vote that 
undergoes a change from $A$ to $B_{i}$ (or $B_{i}$ to $A$ ) is called an anti- $A$ noise contaminated vote (or anti- $B$ noise contaminated vote, respectively).

7. Two types of noise are considered: inhomogeneous noise that influences the votes within an inhomogeneous block (or blocks) of cells and homogeneous noise that is distributed uniformly over the whole nation randomly.

8. A set of anti- $A$ homogeneous noise (or anti- $B$ homogeneous noise) is dispersed uniformly over the nation, producing a uniform chance of converting $\mathrm{A}$ to $B_{i}$ (or $B_{i}$ to $A$ ).

9. A set of anti- $A$-inhomogeneous noise (or anti- $B$-inhomogeneous noise) is defined as the union of non-overlapping rectangle blocks of size $n_{l} \times n_{w}$, on each of which all the votes for $A$ (or $B_{i}$ ) will be changed to $B_{i}$ (or $A$ ). The corresponding union of these rectangular areas is called a noise-inhomogeneous area, and $n_{l} \times n_{w}$ is called the size of noise blocks ${ }^{2}$.

10. In accordance with the above two types of noise, the anti- $A$ noise contaminated votes (or anti- $B$ noise contaminated votes) comprise the two types of contaminated votes depending on the noise type, namely, the anti- $A$ homogeneous-noise contaminated votes (or anti$B$ homogeneous-noise contaminated votes) and anti- $A$-inhomogeneous-noise contaminated votes (or anti- $B$-inhomogeneous-noise contaminated votes).

11. We call a region inhomogeneous-noise contaminated if and only if the intersection of the region and the noise-inhomogeneous area is not empty.

12. Because we are interested in computing the lower bounds of voting stability throughout this paper, we consider only the anti-A noise in the analysis. Thus when we refer to noise, inhomogeneous noise, homogeneous noise, or contaminated votes hereinafter, anti-A noise, anti- $A$-inhomogeneous noise, anti- $A$ homogeneous noise, anti- $A$-noise-contaminated votes are implied.

Definition $1 p \alpha N$ is a homogeneous noise measurement, reflecting the amount of homogeneous noise which causes a change in the voting. Here p refers to the probability of a vote for A changing to a vote for $B_{i}$ in the presence of homogeneous noise when selecting between $A$ and $B_{i}$ for any $i . C \alpha$ is defined as $a$ inhomogeneous noise measurement, reflecting the amount of inhomogeneous noise which causes a change in the voting, where $C$ denotes the total size of noise inhomogeneous areas.

According to Definition 1, the homogeneous noise measurement and the inhomogeneous noise measurement denote the total number of cells that change their votes from $A$ to $B_{i}$ under the pair-wise selecting strategy subjected to homogeneous noise and inhomogeneous noise, respectively.

Assumption We always assume that the voting population of a nation is large and the amount of noise is large, so that both the total number of noise contaminated votes and the size of inhomogeneous noise contaminated area are large.

A region is referred to pro $A$ ( $A$ dominated) or pro $B$ ( $B$ dominated) if $A$ dominates $B$ in the region or if $B$ dominates $A$ respectively.

The pair-wise-best strategy we have adopted in the current model implies that the probability that $A$ wins the election by the poll involving candidates $A, B_{1}, \cdots, B_{m}$, should be equal to the $m$-th power of the probability that $A$ wins when only candidates $A$ and $B_{i}$ are involved.

\section{Theoretical Results}

\subsection{Main Theorems}

\footnotetext{
${ }^{2}$ Intuitively, the "homogeneous noise" is isolated and scattered randomly over discrete "points" of the nation while "inhomogeneous noise" is distributed over connected, continuous areas which may be randomly distributed across the nation.
} 
Definition 2 For any voting systems, $S=\operatorname{Prob}\left(A_{N} \mid A_{F}\right)$ is used to denote the stability of the voting system. Here, $\operatorname{Prob}\left(A_{N}\right)$ and $\operatorname{Prob}\left(A_{F}\right)$ denote, respectively, the probabilities that $A$ wins in a voting (regional or national) in noisy as well as in noise-free environments.

Using Definition 2, $S$ denotes the chance that a voting system retains the original candidate selection in the presence of noise. This definition is in conformity with our basic approach as mentioned in Section 1, that our interest is to elaborate the conditions such that the voting system retains the original candidate selection in the presence of noise. The stability margins are demonstrated by Theorems 1 and 2 below $^{3}$. The proofs for these two theorems are omitted due to space limitations.

Theorem 1 The stability of national voting is

$$
S_{\text {national }}=\left(\frac{\sum_{j=\left\lceil\frac{N}{2}\right\rceil}^{N}\left(\begin{array}{c}
N-C \\
j
\end{array}\right) \alpha^{\prime j} \beta^{\prime N-C-j}}{\sum_{j=\left\lceil\frac{N}{2}\right\rceil}^{N}\left(\begin{array}{c}
N \\
j
\end{array}\right) \alpha^{j} \beta^{N-j}}\right)^{m}
$$

where $\alpha^{\prime}$ and $\beta^{\prime}$ are the percentages of the votes for $A$ and for $B_{i}$ in the presence of homogeneous noise, i.e., $\alpha^{\prime}=\alpha(1-p)$ and $\beta^{\prime}=\beta+\alpha p$.

Theorem 2 The stability of regional voting is

$$
\left(\frac{\sum_{j=\left\lceil\frac{n}{2}\right\rceil}^{n}\left(\begin{array}{c}
n-t_{1} \\
j
\end{array}\right) P_{A}^{\prime j} P_{B}^{\prime n-t_{1}-j}}{\sum_{j=\left\lceil\frac{n}{2}\right\rceil}^{n}\left(\begin{array}{c}
n \\
j
\end{array}\right) P_{A}^{j} P_{B}^{n-j}}\right)^{m}>S_{\text {regional }}>\left(\frac{\sum_{j=\left\lceil\frac{n}{2}\right\rceil}^{n}\left(\begin{array}{c}
n-t_{2} \\
j
\end{array}\right) P_{A}^{\prime j} P_{B}^{\prime n-t_{2}-j}}{\sum_{j=\left\lceil\frac{n}{2}\right\rceil}^{n}\left(\begin{array}{c}
n \\
j
\end{array}\right) P_{A}^{j} P_{B}^{n-j}}\right)^{m}
$$

where

$t_{2}=\frac{C}{n_{l} n_{w}} \cdot\left(\left\lceil\frac{n_{l}-1}{r_{l}}\right\rceil+1\right)\left(\left\lceil\frac{n_{w}-1}{r_{w}}\right\rceil+1\right), t_{1}=\frac{C}{r_{l} r_{w}}, \quad P_{A}=\sum_{y=0}^{\left\lfloor\frac{r_{l} r_{w}}{2}\right\rfloor}\left(\begin{array}{c}r_{l} r_{w} \\ y\end{array}\right) \beta^{y} \alpha^{r_{l} r_{w}-y}$,

$P_{B}=\sum_{y=0}^{\left\lfloor\frac{r_{l} r_{w}}{2}\right\rfloor}\left(\begin{array}{c}r_{l} r_{w} \\ y\end{array}\right) \alpha^{y} \beta^{r_{l} r_{w}-y}, P_{A}^{\prime}=\sum_{y=0}^{\left\lfloor\frac{r_{l} r_{w}}{2}\right\rfloor}\left(\begin{array}{c}r_{l} r_{w} \\ y\end{array}\right) \beta^{\prime y} \alpha^{\prime r_{l} r_{w}-y}, P_{B}^{\prime}=\sum_{y=0}^{\left\lfloor\frac{r_{l} r_{w}}{2}\right\rfloor}\left(\begin{array}{c}r_{l} r_{w} \\ y\end{array}\right) \alpha^{\prime y} \beta^{\prime r_{l} r_{w}-y}$.

\subsection{Theoretical Conclusions and Conjecture}

3.2.1 Conclusions Figure 1(a) shows the stabilities of national and regional voting with different amounts of homogeneous and inhomogeneous noise, when the size of each region is fixed. All these figures when coupled with inequalities 1 and 2 clearly show that, excluding some exceptional cases when the inhomogeneous noise is negligible, regional voting is always more stable than national voting, and when the size of regions increases starting with $1 \times 1$ and ending with the size of the nation, the stability of regional voting first increases, then decreases, starting and ending with that of national voting.

Only in situations where there is very little inhomogeneous noise or when inhomogeneous noise is absent, is national voting more stable than regional voting. This can be seen

\footnotetext{
${ }^{3}$ In Theorem 2, the ceiling operation in calculating $t_{2}$ is used to develop a sufficient condition of stability that constitutes the worst possible condition whereby each of the noise blocks contaminates a maximum number of regions. With a careful analysis, by taking an average of the numbers of regions polluted by all the noise blocks, we can remove the ceiling operation in inequality 2 for estimating the stability of regional voting in an average case.
} 

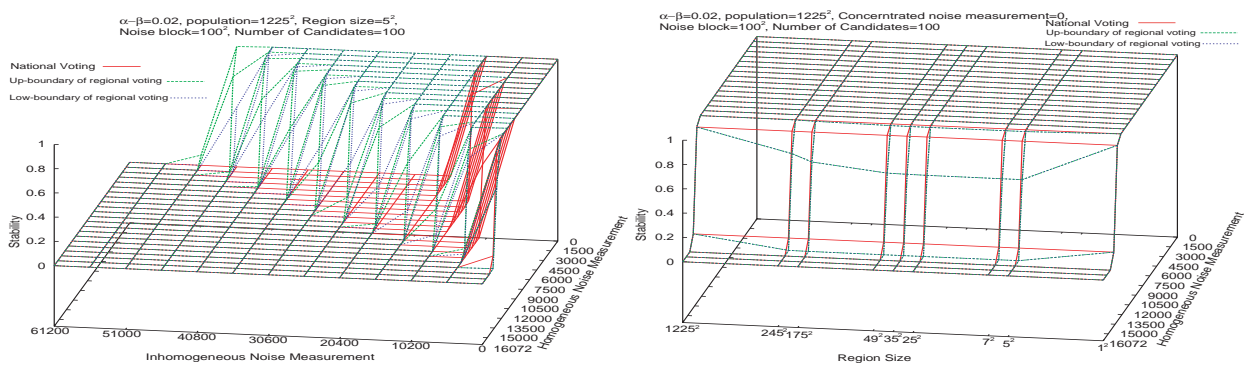

(a) Different amounts of homogeneous noise \& different (b) Different amounts of homogeneous noise \& differamount of inhomogeneous noise, when region size is fixed ent region sizes, when inhomogeneous noise is absent

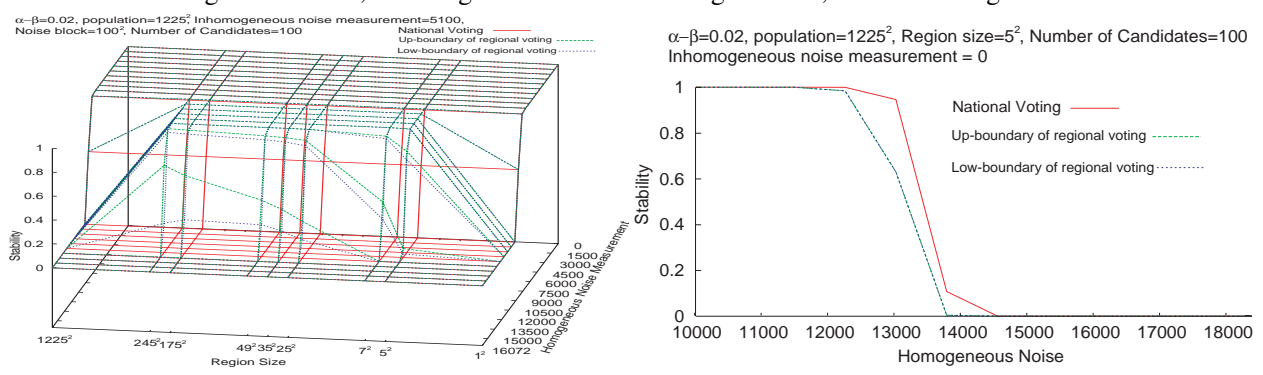

(c) Different amounts of homogeneous noise \& different (d) Different amounts of homogeneous \& inhomogeregion sizes, when some inhomogeneous noise is present neous noise, when region size is fixed.

Figure 1: Stability Diagram of National and Regional voting

from Figure 1(b). It can also be seen from Figure 1(d), which is an enlarged picture of the rightmost section of Figure 1(a).

In summary, our conclusions are: As long as there are certain amounts of inhomogeneous noise present, the regional voting approach always has a better recognition rate / classification accuracy in comparison with national voting; regional voting with medium sized regions is always better than that with small and with large regions. Only in present of great amount of homogeneous noise but without or with only very little inhomogeneous noise may regional voting be not as good as national voting, although we believe this to be to optimistic to have only homogeneous noise existed.

3.2.2 Conjecture We believe that the above conclusion related to national and regional versions of matching remains valid even when the decision making process involves complicated matching schemes such as feature extraction by the PCA method.

\section{Experiments on Face Recognition}

4.1 Experiment on FERET face database We use the PCA approach (with Mahalanobis Cosine measurement) as the national matching scheme, and also as the way to determine the winner in each region in regional matching schemes.

We use the standard FERET face database [ $[\boldsymbol{\theta}]$ : "fa" is the set of gallery images containing 1196 grayscale images, "fb", "fc", "dup1" and "dup2" are sets of probe images. The faces are firstly normalized into standard format: each image is rotated, scaled and cropped to a size $150 \times 130$ pixels ( 150 per column, 130 per row), so that the distance between the eye centers is 56 pixels and the line between eyes lies on the 53rd pixel from top. ${ }^{4}$

\footnotetext{
${ }^{4}$ The recognition rates may vary slightly if we change the eye distances and eye positions in the picture.
} 
The standard CSU training set ${ }^{5}$ consisting of 501 faces are used for training: for national voting, these 501 faces are used to generate the eigen vectors and eigen values; for regional voting, the windows in corresponding faces are used to generate the eigenvectors and eigen values. During eigen-face decomposition, we remove first two eigen-values and the corresponding eigenvectors, then the first $k(k=40 \% \times \min (M, N)$ in our experiments, where $M, N$ represents the number of effective pixels in each image and the number of training images, which is 501 here) of the remaining eigen values and the corresponding eigenvectors are used for pattern projection and matching.

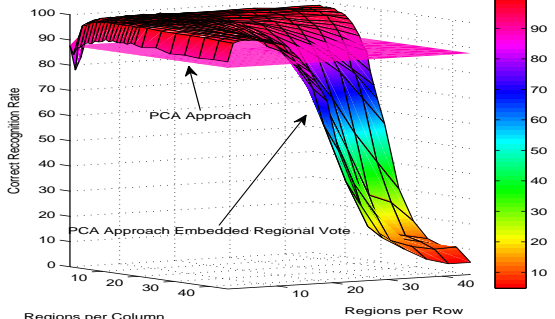

(a) "fb"

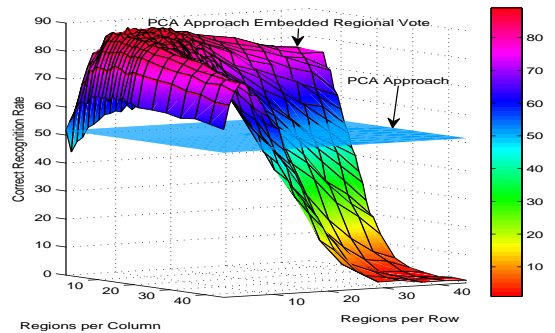

(c) "dup1"

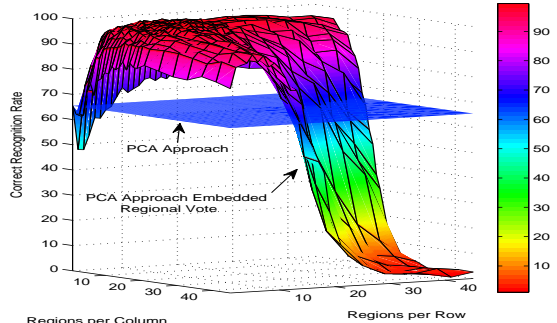

(b) "fc"

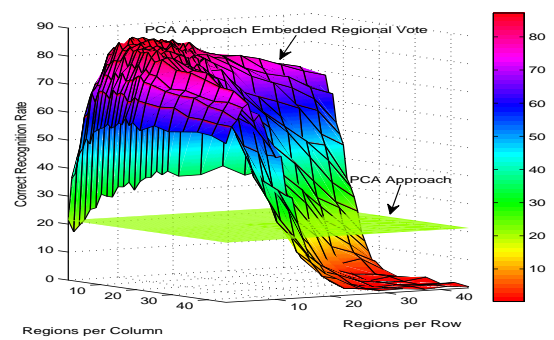

(d) "dup2"

Figure 2: The Recognition Rates on FERET sets with different number of windows

Due to the known alignment problem ([ष]) for face recognition, we allow the perturbing of images in a neighborhood during the matching process: When computing the similarity between a region/window of a probe image and a region/window of a gallery image for regional matching scheme, we perturb the position of the window at most 3 pixels in each direction, and limit the total perturbing to 4 pixels. Then, over the gallery image, compute the similarities of the gallery sub-image in all nearby positions with the probe sub-image, and select the largest among these candidate similarities as the similarity distance. Consequently, when computing the similarity between a gallery and a probe image for the national matching model, we locate the windows of size $146 \times 126$ in the gallery image and the probe image, allowing the window in the gallery image to be perturbed as above mentioned, compute the similarities between each such window in the gallery image and the window centered in the probe image. Among these many similarity values, we select the largest as the similarity between the probe and the gallery image. ${ }^{6}$ The standard ellipse mask included in the FERET data collection is also applied to remove the back ground and hair corners. ${ }^{7}$

\footnotetext{
${ }^{5}$ http://www.cs.colostate.edu/evalfacerec/data.html

${ }^{6}$ When we partition an image into windows, we leave a bar of 3 pixels width on the boundaries so that all the windows can be perturbed in all directions.

${ }^{7}$ However, since the gallery images are perturbed for reliable alignment, the mask is only applied for the images
} 
We compare the recognition performances of national vote/direct popular vote and regional vote/Electoral College schemes. ${ }^{8}$ Figures 2(a), 2(b), 2(c) and 2(d) show the recognition rates with different numbers of regions/windows (when the image is partitioned into $x$ rows and $y$ columns, there are $x y$ windows/regions). They show that the regional matching works much better than the national matching scheme as long as the size of windows is neither too small not too big. The best recognition accuracies for "fb", "fc", "dup1" and "dup2" are $99.42 \%, 99.485 \%, 89.06 \%$ and $87.215 \%$ respectively. ${ }^{9}$

It is easy to see that the regional voting embedded approach has a marked improvement over the national voting type recognition algorithm for face recognition.

4.2 Experiments on Yale face dataset The system for the experiments on the Yale Face datase ${ }^{10}$ is set-up in almost the same way as above with following exceptions:

(1) The gallery consists of 45 face images (15 persons, each with 3 images: centerlight, noglasses, and normal);

(2) We choose the face images named glasses, happy, leftlight, rightlight, sad, sleepy, surprised, and wink as the probe set;

(3) We use all the 45 images in the gallery set as the training set (Notice that we do not use the identities of the faces in the training set);

(4) In eigen-face decomposition, the number of eigen faces we choose is $k, k=75 \% \times$ $\min (M, N)$, where $M, N$ represents the number of number of effective pixels in each image and the number of training images, which is 75 here. ${ }^{11}$

(5) For any image or sub-image of a probe face $p$, We take it as a vote for a face $g$ if the similarity of the image (or sub-image) with any one of three faces of $g$ is the largest among the similarities of this image (or sub-image) with all the images (or sub-images) in the gallery.

The result is shown in Figure 3. The best recognition accuracy is $100 \% .^{12}$

in the windows for direct popular matching; the part of the mask is only applied for the sub-images in the windows, rather than the entire image, for Electoral College matching.

${ }^{8}$ If there is a tie among $t$ gallery images before the final decision is made and one of them is the correct answer, we only count $1 / t$ when we calculate the accuracy. We believe that this is a fair strategy.

${ }^{9}$ It's also observed that when the numbers of regions per row and the numbers of regions per columns are within the range of 8 and 12, the minimal accuracies for "fb", "fc", "dup1" and "dup2" are 98.862\%, 96.735\%, 85.474\% and $82.353 \%$ respectively.

For reference purposes, although it is not the main purpose of this paper, the known best results can be found in [ $\square$ ]: fb and fc: $99.5 \%$, dup1: $85.0 \%$ ([四), dup2: $79.5 \%$. We should note here that the size of images in [] is $203 \times 251$, which is significantly larger than ours; and it is known that the image size affects recognition performances (and usually higher resolution (as long as not too large) images have better identification rates).

${ }^{10} \mathrm{http} / / \mathrm{cvc}$.yale.edu/projects/yalefaces/yalefaces.html. The eye location values of Yale face dataset is downloaded from http://vismod.media.mit.edu/vismod /classes/mas622-00/datasets/. A few eye location data were swapped among different faces by mistake - and it is easy to find and correct.

${ }^{11}$ We use $k=75 \% \times \min (M, N)$ instead of $k=40 \% \times \min (M, N)$ which is used in the experiments for FERET, simply because the training set size $N$ is so small here.

${ }^{12}$ It is also observed that, when the numbers of rows and columns that the images are partitioned into are within the range of 8 and 12, the minimal accuracy is $99.169 \%$.

Note that, it is not our purpose to compare our results with others; and although the Yale face dataset has been widely used in academic research, it is hard to compare the results since different researchers conducted the experiments with different partitionings and therefore tested on different subsets with different galleries. For reference purposes, we summarize some of the results here: Using leave-one-out method (leave one image per person each time for testing) [四] reported the best accuracy of was $89.09 \%$. When a training set containing six images per person, and the rest for testing, [ $\mathrm{\theta}$ ] reports an accuracy of $88.7 \%$. When five images, namely center light, glasses, happy, leftlight and noglasses, of each subject are used to train a classifier, the rest were used for test, the best report was reported in [四] with an accuracy of $94.44 \%$. When the first five images of each subject are used as training samples, the others are taken as test samples, [四] reports an accuracy of $88.9 \%$. 


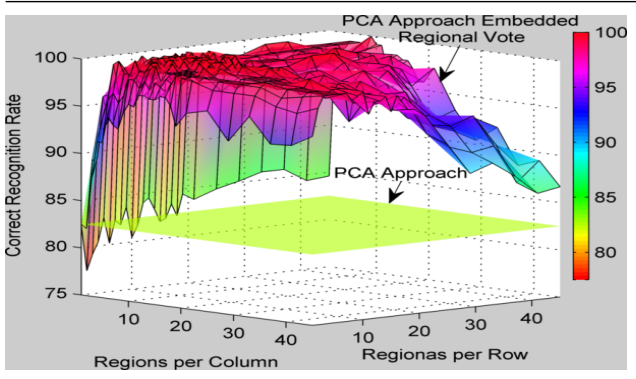

Figure 3: The Recognition Rates for Yale Face Dataset with different number of windows

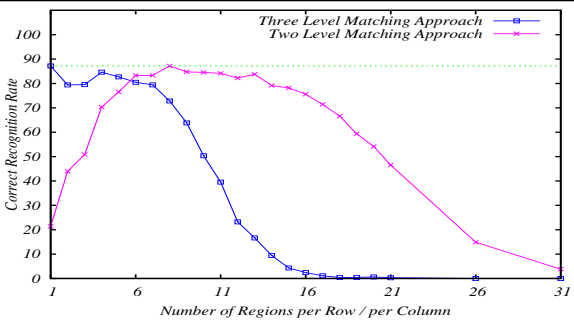

Figure 4: 2- \& 3- Level Regional Voting Experiments on FERET Dup2 using PCA as Base Matching Approach

\section{Discussions, Conclusions and Further Work}

\subsection{Major difference of the contributions of this paper from that of previous works}

1. While there are methods that uses patches (as opposed to full image classification) for face recognition(e.g., Modular Eigen-Templates of [ [ $]$ ]), we do not select patches in specific areas, while other techniques, such as $[\boldsymbol{[}]$, detect and use only patches in specific areas.

2. In our theory, regional vote is a "regional" version of a national approach. In the context of face recognition, approaches such as LBP and local gabor patterns [ $\square]$ seem to be very close to our regional vote scheme. However, none of them has a corresponding national version. In other words, they are not the regional versions of any national vote/holistic approaches.

5.2 3+ Level Regional Voting A natural and immediate question shall be: how about three or more level regional voting system? We take the regional voting we discussed above as two level regional voting; three level regional voting further divides each region into subregions in each of which the basic matching takes place, simple majority rule applies in each region according to numbers of winning subregions and then in the nation according to numbers of winning regions. Simulations and experiments show that: for the situation when two level voting reaches its best performance, three level cannot do any better; when the regions are too big for two level voting to reach its best, three level voting can improve the performance with "best" subregion sizes up to the level of the best of two level voting. This can be illustrated by the example shown in Figure 4, where we let the numbers of regions per row and per column be equivalent, the numbers of subregions per row and per column in a region also be equivalent. The curve of three level approach shows the best results of three level approach for any fixed two level region partitions. ${ }^{13}$ Therefore, $3+$ regional level voting is not necessary for the purpose of accuracy improvement; however, it can change the way to reach a "best" performance: we start with "large" region sizes, then seek best performance by finding proper lower level subregion sizes or probably by further dividing subregions into even lower level sub-subregions, $\cdots$, to establish a 4 or more level voting system.

5.3 Conclusions and Further Work We have shown that, for multi-candidate matching, regional voting is always more stable than national voting, and regional voting with medium sized regions is the best, when both homogeneous noise and inhomogeneous noise exist. Experiments showed the correctness of the theory.

This paper models inhomogeneous noise as blocks of noise, under the influence of which all the voters/cells for one candidate will change their votes. In real applications, it may be possible that only a certain portion of voters under inhomogeneous noise will change the votes. It should be interesting to develop models that are able to deal with such situations.

\footnotetext{
${ }^{13}$ Note that, when region number equals 1 , two level and three level regional voting are indeed equivalent to national voting and two level regional voting, respectively.
} 
In real applications, we have no idea what the noise consists of in most cases. Intuitively, noise can be either "localized" as inhomogeneous noise, or "well distributed" as homogeneous noise. Are there any other types of noise? It is an interesting topic to investigate.

The nation is assumed to be large in our model (see Section 2). In the applications, such as face recognition, $100 \times 100$ seems to be "large", however we really don't have a precise definition of "large". Further investigation should be important.

Acknowledgements: This research is supported by a Discovery Grant of NSERC, Canada. Portions of this research of this paper use the FERET database of facial images collected under the FERET program. The author wishes to thank the anonymous reviewers for their helpful comments.

\section{References}

[1] T. Ahonen, A. Hadid, and M. Pietikäinen. Face recognition with local binary patterns. In Proc. of 9th European Conf. on Computer Vision, pages 469-481, May 7-13 2004.

[2] M. J. Black and P. Anandan. The robust estimation of multiple motions: Parametric and piecewise-smooth flow fields. Computer Vision and Image Understanding, 63(1): 75-104, 1996.

[3] L. Chen and N. Tokuda. Regional voting versus national voting -stability of regional voting (extended abstract). In Int. ICSC Symposium on Advances in Intelligent Data Analysis, Rochester, New York, USA, June 22-25 1999.

[4] L. Chen and N. Tokuda. A general stability analysis on regional and national voting schemes against noise - why is an electoral college more stable than a direct popular election? Artificial Intelligence, 163(1):47-66, 2005.

[5] L. Chen, W. Xue, and N. Tokuda. Classification of 2-dimensional array patterns: Assembling many small neural networks is better than using a large one. Neural Networks, 23(6):770-781, August 2010.

[6] X. He, S. Yan, Y. Hu, P. Niyogi, and H. Zhang. Face recognition using laplacianfaces. IEEE Trans. Pattern Analysis \& Machine Ingelligence, 27(3):328-340, MARCH 2005.

[7] N. Herbert. Quantum Reality. Anchor Books, New York, 1985.

[8] B. Moghaddam and A. Pentland. Probabilistic visual learning for object representation. IEEE Trans. Pattern Analysis \& Machine Intelligence, 19(7):696 - 710, July 1997.

[9] P. Phillips, H. Wechsler, J. Huang, and P. Rauss. The FERET database and evaluation procedure for face recognition algorithms. Image and Vision Computing, 16(5):295306, 1998.

[10] C. Schmid and R. Mohr. Local grayvalue invariants for image retrieval. IEEE Trans. Pattern Analysis \& Machine Intelligence, 19(5):530-535, 1997.

[11] K. Tan and S. Chen. Adaptively weighted sub-pattern pca for face recognition. Neurocomputing, 64:505-511, 2005. 
[12] P. Wang, L. C. Tran, and Q. Ji. Improving face recognition by online image alignment. In Proc. of 18th Int. Conf. on Pattern Recognition, volume 1, pages 311-314, Hong Kong, Aug. 20-24 2006.

[13] S. Xie, S. Shan, X. Chen, X. Meng, and W. Gao. Learned local gabor patterns for face representation and recognition. Signal Processing, 89(12):2333-2344, 2009.

[14] Y. Xu, J. Yang, J. Lu, and D. Yu. An efficient renovation on kernel fisher discriminant analysis and face recognition experiments. Pattern Recognition, 37:2091-2094, 2004.

[15] J. Zou, Q. Ji, and G. Nagy. A comparative study of local matching approach for face recognition. IEEE Transactions on Image Processing, 16(10):2617-2628, 2007. 